Classification

Physics Abstracts

$64.70 \mathrm{E}-61.30$

\title{
Tilt susceptibility and bare correlation length at a smectic-A-smectic-C phase transition
}

\author{
Y. Galerne \\ Laboratoire de Physique des Solides (*), \\ Université de Paris-Sud, Bât. 510, 91405 Orșay, France \\ (Reçu le 17 janvier 1983, révisé le 18 avril, accepté le 26 avril 1983)
}

\begin{abstract}
Résumé. - Nous présenterons nos résultats de mesures de la susceptibilité d'inclinaison à la transition smectique-A-smectique-C du azoxy-di( $\alpha$-méthylcinnamate de undécyl)-4,4'. Nous en déduisons l'exposant critique de la transition $\gamma=1,28 \pm 0,03$, et la longueur de cohérence nue $\xi_{0} \sim 2-5 \AA$. Ces résultats sont cohérents, selon le critère de Ginzburg, avec un comportement non classique à la transition.
\end{abstract}

Abstract. - We report measurements and data analysis of the tilt susceptibility at the Sm-A-Sm-C transition of azoxy-4,4'-di-undecyl- $\alpha$-methylcinnamate. We deduce the critical exponent of the transition $\gamma=1.28 \pm 0.03$ and the bare correlation length $\xi_{0} \sim 2-5 \AA$. These results are consistent through the Ginzburg criterion, with a non-classical behaviour at the transition.

Both the smectic-A and smectic-C phases of liquid crystals are layered systems. In the A-phase, the molecules are, statistically speaking,packed perpendicularly to the layers, while in the C-phase, they are tilted through an angle $\psi$. This angle $\psi$, together with the azimuthal angle $\phi$ (Fig. $1 a)$, defines the tilt direction in the C-phase, leading to the choice of $\psi \mathrm{e}^{i \phi}$ as the order parameter of the A-C transition [1]. Therefore, if one may neglect the effects of anisotropies and the LandauPeierls instability of the layers [2], the A-C transition should belong to the same universality class as the $3 \mathrm{D}-X Y$ model and superfluid helium [3]. The A-C transition should thus behave like the fluid-superfluid transition in helium, with the same critical exponents, except for far enough away from the transition for the Ginzburg criterion to predict classical behaviour [4], i.e. for

$$
\frac{\Delta T}{T_{\mathrm{c}}}>\frac{k_{\mathrm{B}}^{2}}{32 \pi^{2}\left(\Delta C_{\mathrm{p}}\right)^{2} \xi_{0}^{6}},
$$

where $\Delta C_{\mathrm{p}}$ is the mean-field heat-capacity jump and $\xi_{0}$ is the bare correlation length. The temperature $\left(\Delta T=T-T_{\mathrm{c}}\right)$ of the Ginzburg crossover is controversial and two sorts of behaviour, classical and helium-like, are widely reported in the literature [5-14]. In this paper, we present results of tilt susceptibility and bare correlation length measurements which try to clarify the question. The measurements of tilt susceptibility were performed using a magnetic field as an

(*) Laboratoire associé au C.N.R.S. 


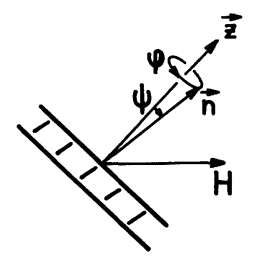

(a)

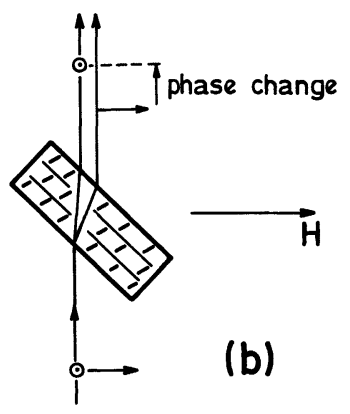

Fig. $1 a .-\psi$ is the angle between the normal to the layers $\mathbf{z}$ and the director $\mathbf{n}$. The azimuthal angle $\phi$ specifies the direction of the director.

Fig. $1 b$. - The laser light splits into two rays of orthogonal polarizations inside the sample. The phase change between them is measured and yields the tilt angle $\psi$.

external force, the induced tilt being detected by an interferometric method [14]. In this way, we have obtained in the smectic-A phase of undecylazoxymethyl-cinnamate (AMC-11), what we believe to be the first direct and absolute determination of the tilt susceptibility. From these measurements, we deduce $\xi_{0}$ the bare correlation length, and $\gamma$ the critical exponent of the susceptibility with temperature; these two results having to be consistent through the Ginzburg criterion.

\section{Experimental.}

The sample, set-up and experimental methods are the same as described in reference [14].

1.1 SAMPLE. - The smectic compound used is undecylazoxymethyl-cinnamate (AMC-11), purified several times in hexane. The main advantages of this material are its weak chemical degradation when enclosed in sealed cells (with a drift of $T_{c}$, the transition temperature, slower than $10^{-3} \mathrm{~K} / \mathrm{h}$ ), and the almost perfect samples that it gives in the homeotropic alignment when the glass plates are treated with silane [15]. A lot of samples were prepared in this way; the thickness of the sample is given by a Mylar spacer, and is measured to a precision of $2 \mu \mathrm{m}$ by direct use of a Palmer micrometer on the solid AMC-11, after the sample is dismounted.

1.2 Temperature CONTROL. - The sample is placed inside a two-stage oven. In each stage, the temperature is detected with a platinum resistor and thermocontrolled by independent circuits. This system keeps the temperature of the sample stable to better than $10^{-3} \mathrm{~K}$ in space and time, as this is directly checked by observing the A-C transition itself, or by using a third independent platinum thermometer in place of the sample.

1.3 MAGNETIC-FIELD PERTURBations OF THE TEMPERATURe-CONTROL SYSTEM. - The sample is submitted to a magnetic field $\mathrm{H}$ oriented at $45^{\circ}$ with respect to the normal to the smectic layers (Fig. 1). This configuration provides a strong coupling to the order parameter $\psi$, and thus allows precise measurements of the susceptibility in the smectic-A phase. The magnetic field is produced by a classical electromagnet and may reach up to $16 \mathrm{kOe}$. In addition to its action on the director of the smectic, the magnetic field perturbed the platinum resistor probe, causing effects that had to be taken into account. One kind of perturbation is due to the magnetoresistance of platinum; 
it makes a small correction to the temperature ( $\sim 1 \mathrm{mK}$ in a $12 \mathrm{kOe}$-magnetic field). Another but stronger perturbation can arise from vibrations of the wires due to Laplace's forces acting on the measuring and alternating $(1 \mathrm{kHz})$ currents. This effect results in an energy dissipation which looks like magnetoresistance to the measuring bridge. In order to avoid such problems all the measuring wires lying in the high magnetic field area had to be rigidified; this was mainly done by using glue.

1.4 OPTICAL INTERFERENCE. - The measurement of the induced tilt angle $\psi(H)$ is done using an interferometric method [14]. A He-Ne laser beam passing through the sample divides into two rays of orthogonal polarizations (ordinary and extraordinary polarizations) which follow different paths and exhibit a phase difference outside the sample (Fig. 1b). Then, a Soleil compensator, adjusted to give the appropriate phase compensation, restores the initial polarization. This is checked by placing sample and compensator between crossed polarizers and verifying the total extinction of the light outside the system. In order to equalize the light between the two rays, the initial polarization was chosen to be $45^{\circ}$ with respect to the plane containing the magnetic field and the director (plane of Fig. 1). The incident angle of the laser beam is also chosen to be $45^{\circ}$. In this way, there is a strong first-order coupling of the phase change with the tilt angle, resulting in an accurate measurement of the tilts $\left(\delta \psi \sim 3 \times 10^{-4} \mathrm{rad}\right.$. for an error of $\lambda / 50$ in the adjustment of the compensator). $\psi$ is then determined from an exact calculation [16] taking into account the measured phase change, the sample thickness, and the thermal variations of the optical indices (measured previously [17]). In addition to the precision, another advantage of this optical method of measuring $\psi$, is to permit permanent checks of the alignment quality of the sample, just by looking at its optical image. In this way, we made sure that we did not measure ultra-precisely integrated quantities on disoriented samples, which would be of lesser physical interest.

\section{Analysis of the data.}

For each sample, we made a series of measurements of the induced tilt angle at different temperatures and magnetic fields. When measuring these three variables $(\psi, T, H)$, we get an experimental description of the equation of state. In this paper, we restrict our study to the smectic-A phase; therefore, the small- $\psi$ (or weak-field) approximation is possible, and the equation of state can be expanded as a power series in $\psi$ near the critical isochore in the form [18] :

$$
\frac{1}{2} \chi_{a} H^{2}=a(T) \psi+b(T) \psi^{3},
$$

where $a(T)$ and $b(T)$ are coefficients which are power functions of $\Delta T=T-T_{\mathrm{c}}$, with exponents $\gamma$ and $\gamma-2 \beta$, respectively. $a(T)$, also called $B_{\perp}$ [5] or $D$ [8], can be understood as the inverse of the tilt susceptibility in a magnetic field. $b(T)$ accounts for the curvature of the isotherms which appears in the $\mathrm{mK}$-range of the transition.

2.1 ISOTHERM ANALYSIS. - The above expansion makes it possible to analyse the data by isotherms, using an independent least-squares fit to equation 2 for each temperature. Such fits (each to about 20 experimental points) are usually good to within the experimental uncertainties. However anomalies, such as bad fits and hysteresis, appear in the thin sample measurements. These anomalies, which we do not consider here, can be interpreted in terms of a constraint field produced by an extra (or a missing) layer in the sample [14, 16]. The compression of the sample is then inversely proportional to its thickness, and may thus be neglected in thick enough samples. In this paper, we only consider the measurements obtained from samples thicker than $250 \mu \mathrm{m}$, where the constraint anomalies are practically invisible. In this case, the least-squares 
fit to equation 2, applied on a set of about 20 experimental data, yields $a(T)$ to an accuracy better by a factor of 4 to 5 than the accuracy calculated on each direct measurement (for instance, the temperature error in the determination of $a(T)$ is decreased to $\left.\delta T \sim 2 \times 10^{-4} \mathrm{~K}\right)$.

Thus, the analysis of the isotherms yields a series of values for $a(T)$, i.e. the measurement of the temperature dependence of the inverse of the tilt susceptibility. From these $a(T)$-series, we extract the following information. We first get a direct estimate of the transition temperature $T_{\mathrm{c}}$ in each $a(T)$-series, by extrapolating to $a\left(T_{\mathrm{c}}\right)=0$. This determination of $T_{\mathrm{c}}$ is approximate. We only use it to measure the drift of the transition with time, and to make a primary estimate of $T_{\mathrm{c}}$ at any moment of the experiment. We then deduce quantities of greater physical interest such as the critical exponent $\gamma$ and the bare correlation length $\xi_{0}$ (following paragraphs).

\subsection{ASYMPTOTIC BEHAVIOUR OF THE TILT SUSCEPTIBILITY.}

2.2.1 Least-squares fits. - The asymptotic dependence of the tilt susceptibility on temperature is supposed to obey a power law with critical exponent $\gamma$; i.e. :

$$
a(T)=\bar{a}\left(T-T_{\mathrm{c}}\right)^{\gamma} \text {. }
$$

In order to determine $\bar{a}$ and $\gamma$, we could run a three parameters least-squares fit on our experimental data $a(T)$, but because of the coupling which appears in such a fit between the parameter $T_{\mathrm{c}}$ and the saturation of $a(T)$, we have adopted another process of analysis. We first take a trial value for $T_{\mathrm{c}}$ in the range of the primary estimate of $T_{\mathrm{c}}$ (see previous paragraph). Then, after having eliminated the points corresponding to a higher temperature than $T_{1}$ (some cut-off temperature which will be varied later), we fit a power law to the remaining data. Practically, this is done using a linẹar least-squares fit on the two parameters $: \log a, \log \left(T-T_{\mathrm{c}}\right)$, a correct weighting of the data (proportional to $\Delta T^{2}$ ) being used near the transition to allow for the constant error in temperature. The result of such an analysis is shown in figure $2 a$ for a series $a(T)$ measured on a sample of $340 \mu \mathrm{m}$-thickness, and in figure $3 a$ for another typical series obtained on a $335 \mu \mathrm{m}$-thick sample. The solid lines in these figures correspond to the least-squares fit applied to the data represented by dots, for which the temperature is smaller than $T_{1}$. The arrows joining figure $2 a$ to $2 b$ and figure $3 a$ to $3 b$, show the results of the corresponding fits $: \gamma\left(T_{1}\right)$ and its standard deviation.

2.2.2 Stability of the fit. $-T_{1}$ is then changed to other values and the previous fit to equation 3 is repeated. In this way, we get a series of results $\gamma\left(T_{1}\right)$ with their associated standard deviations, which we plot in figure $2 b$ (and $3 b$ ). This procedure is similar to that of references [19] and [14]; it allows deviations from the power law to be detected and $T_{\mathrm{c}}$ to be found.

Except farther from the transition than the dashed line, where some decrease of $\gamma\left(T_{1}\right)$ appears, figure $2 b$ and $3 b$ demonstrate a correct stability of the fits. This ensures that nearer to the transition, no deviations from the power law occur due to systematic errors (always hypothetical) or any physical effect. Therefore, the dashed line marks the limit of the power-law behaviour of the $a(T)$-measurements ; beyond it, the data have to be rejected out of the transitional analysis. Farther from the dashed limit, the decrease of $\gamma\left(T_{1}\right)$ indicates some saturation of $a(T)$ (cf. $\left.\S 3.1 .2\right)$.

The use of a similar procedure on the points near the transition yields $T_{c}$. The plot of $\gamma\left(T_{1}\right)$ is repeated for two values of $T_{c}$, separated by only $3 \times 10^{-4} \mathrm{~K}$. When the choice of $T_{\mathrm{c}}$ is correct, each of these values corresponds to opposite divergences of the $\gamma\left(T_{1}\right)$-plot near the transition. As figures $2 b$ and $3 b$ show, we get in this way, reasonable limits on $T_{\mathrm{c}}$. Such good precision in the determination of $T_{\mathrm{c}}$ is similar to that which was obtained in the smectic-C measurements [14]; it results from the statistics involved when fitting a lot of experimental data. Here, in the series of figures 2 and 3, we have about 200 independent measurements (20 in each isotherm fit to Eq. 2).

Between the decrease far from $T_{\mathrm{c}}$ and the divergence near $T_{\mathrm{c}}$, we find a stable plateau in the $\gamma\left(T_{1}\right)$-plots, which yields $\gamma$. 


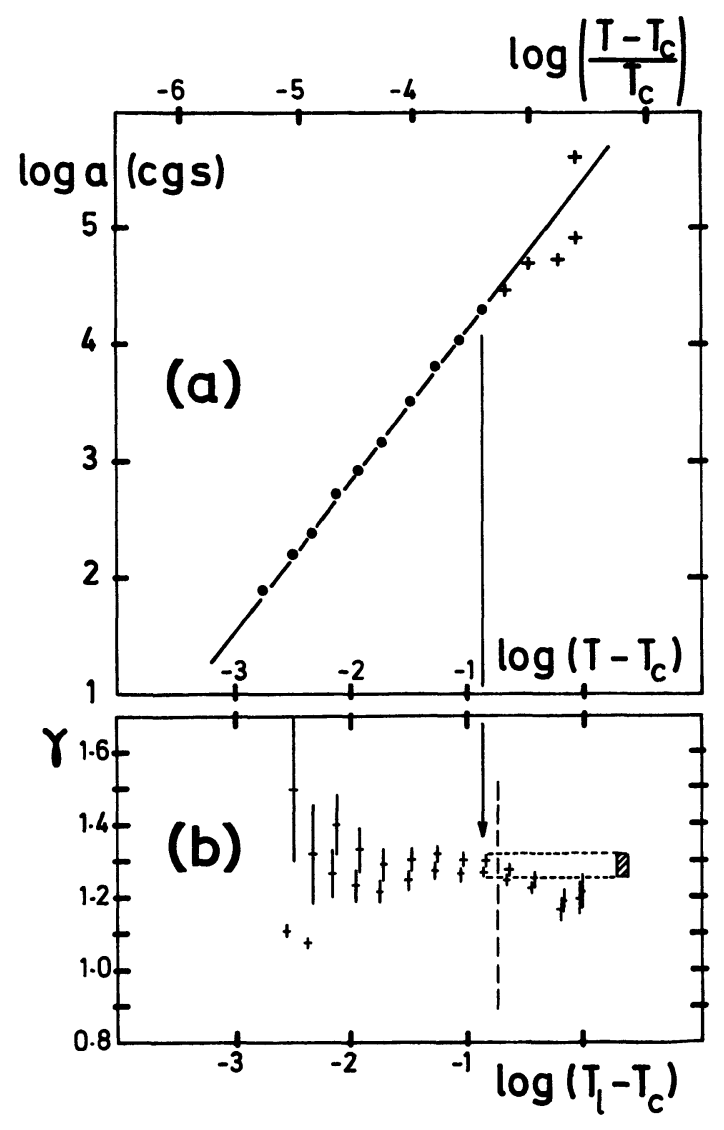

Fig. 2a. - Decimal Log-log plot of a series of $a\left(T-T_{\mathrm{c}}\right)$-measurements obtained on a $340 \mu \mathrm{m}$-thick sample, and a least squares fit (solid line) not including the points marked by crosses (for which $T>T_{1}$ ).

Fig. 2b. - Plot of the result of the previous fitting ( $\gamma$ and its error bar) as a function of the limit temperature $T_{1}$ above which the points are not included in the fit. This stability test shows that some decrease of $\gamma\left(T_{1}\right)$, due to saturation in $a(T)$, occurs at a temperature marked by the dashed line. Two different values of $T_{c}$, slightly different from the good one, are tried. They give plots of opposite divergences near the transition. Between the regions affected by saturation or sensitive to the choice of $T_{c}$, we have a stable plateau which yields $\gamma=1.28 \pm 0.03$.

\section{Physical results and discussion.}

\subsection{DETERMINATION OF $\gamma$.}

3.1.1 Results for $\gamma$. - As discussed in the previous paragraph, the least-squares fits on the power law (1) appear to be stable in the middle range of figures $2 b$ and $3 b$, between the regions affected by saturation or sensitive to the choice of $T_{\mathrm{c}}$. In those measurements, the plateau is wide enough to yield $\gamma$ to within a small error bar $\Delta \gamma$, which contains however the uncertainty in the determination of $T_{\mathrm{c}}$. From the series of figures 2 and 3, we find the non-classical results :

and

$$
\gamma=1.28 \pm 0.03
$$

$$
\gamma=1.31 \pm 0.05, \text { respectively }
$$




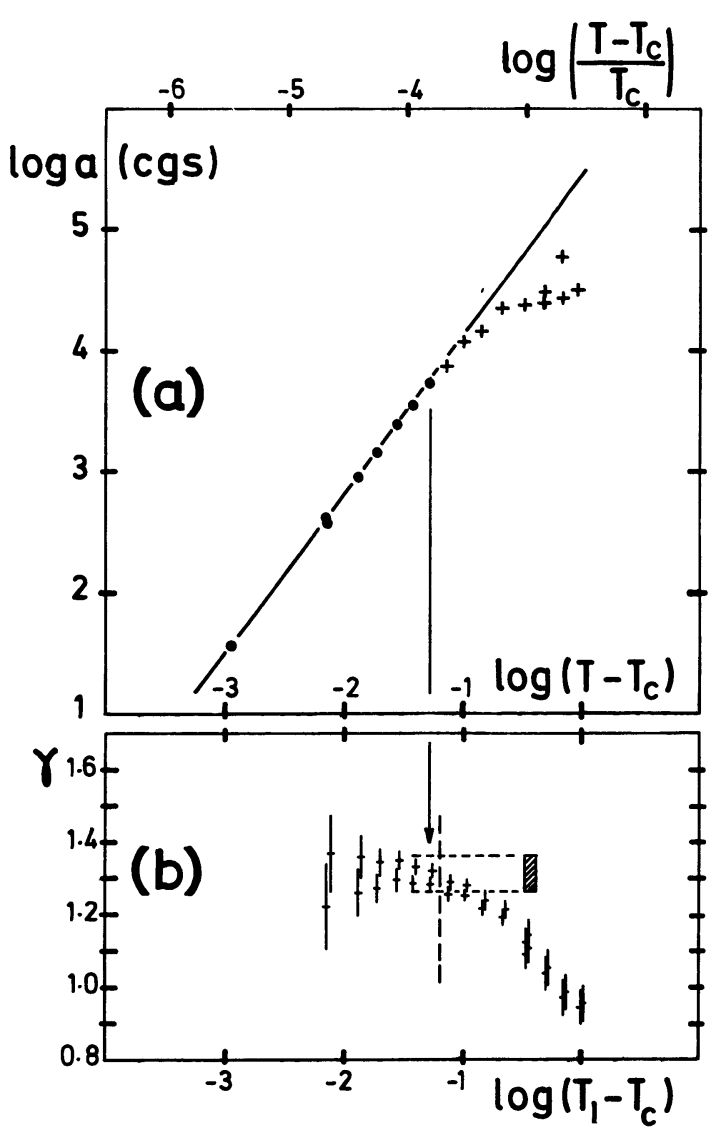

Fig. 3. - Similar to figure 2 , for a $335 \mu \mathrm{m}$-thick sample. In this case, the saturation has a more obvious effect. From this measurement, we deduce $\gamma=1.31 \pm 0.05$.

These results, together with the measurements which are good enough to allow the previous data analysis, are consistent with the value $\gamma=1.28 \pm 0.03$; but generally our series of measurements do not give any result. Because of large saturation, especially in the thick samples $(\S 3.1 .2)$, the saturation region in $\gamma\left(T_{1}\right)$ joins the domain where $\gamma\left(T_{1}\right)$ is sensitive to the choice of $T_{\mathrm{c}}$. In these unfortunate cases, the stable plateau disappears, and makes it impossible to determine $\gamma$. This problem occurs in the thick samples, while compression and the consecutive anomalies $(\$ 2.1)$ affect the thin samples. Therefore, the only results we can get are on the samples of thickness around $300 \mu \mathrm{m}$.

3.1.2 Origin of saturation in $a(T)$. - In contrast to the saturation of the order parameter of smectic-C [14], the saturation of the susceptibility measured in smectic-A far from the transition, is somewhat surprising; it occurs at very small tilt angles for which saturation would seem, at first sight, to be impossible. In order to explain this saturation, we can imagine that the layers of the smectic tilt under magnetic field action, adding to the tilt of the molecules inside the layers. It should result in a systematic increase of the measured tilt-susceptibility, i. e. in the saturation of $a(T)$. This tilt of the layers could occur via a migration of edge-dislocations; those of positive Burger's vector moving towards one plate, the negative ones moving towards the other plate. But such a movement (or glide) of the edge-dislocations needs energy for breaking the layers, or time if it is due to a viscous motion of screw-dislocations [20]. Both mechanisms, if they exist, 
should have negligible amplitude, since we observe that the samples immediately recover the zero-tilt configuration when the magnetic field is switched off. Therefore, the tilt of the layers cannot cause signifiant modifications of the measured values of $a(T)$, nor explain its saturation.

So, we suggest that the saturation of $a(T)$ may be interpreted as a pretransitional effect of the isotropic phase. In the isotropic phase, the smectic planes vanish, and the tilt susceptibility disappears; therefore, although the smectic-isotropic transition is first order, its pretransitional effects may soften the layers near the A-C transition, resulting in a deviation from the $a(T)$ fast-increasing law. This deviation is then analysed as the beginning of a saturation, the amplitude of it depending on the proximity of the isotropic phase, and on the degree of chemical degradation of the sample. In some bad cases then, the isotropic phase occurs just above the A-C transition, giving rise to a large perturbation (cf. Fig. 3).

3. 2 Determination of THE BARE CORRElation LENGTH. - From our data analysis (Figs. 2 and 3), we obtain also the calibration of the power law (3) :

$$
\bar{a}=2.7 \times 10^{5} \pm 0.15 \times 10^{5} \mathrm{cgs} .
$$

Assuming the mean-value of the Frank elastic constants $K \sim 5 \times 10^{-7}$ dyne [1], we estimate the correlation length in the C-phase :

$$
\xi=\left|\frac{K}{2 a}\right|^{1 / 2} \sim 100 \Delta T^{-2 / 3} \AA .
$$

By extrapolation to 0-Kelvin, we obtain a first estimate of the bare correlation length :

$$
\xi_{0}=2.3 \AA \text {. }
$$

But, due to the Ginzburg criterion, the behaviour of the transition should become classical before reaching the 0 -Kelvin temperature. This classical behaviour may start from $\Delta T \sim 1 \mathrm{~K}$, which is approximately the temperature of our last correct measurement (Fig. 2). Under this hypothesis, the correlation length extrapolates to 0-Kelvin with the classical behaviour :

$$
\xi \sim 100 \Delta T^{-1 / 2} \AA \text {, }
$$

and we get :

$$
\xi_{0}=5.3 \AA \text {. }
$$

As can be checked, each of these hypotheses is consistent with the Ginzburg criterion. A calorimetric measurement on a DSC-2 Perking Elmer gives $\Delta C_{\mathrm{p}}=0.5 \times 10^{6} \mathrm{erg} / \mathrm{cm}^{3} \mathrm{~K}$ in AMC-11 (in good agreement with the other $\Delta C_{\mathrm{p}}$-measurements at the $\mathrm{A}-\mathrm{C}$ transition in other compounds [21]). It places the Ginzburg limit at $150 \mathrm{~K}$ from the A-C transition in the first case, and at $1 \mathrm{~K}$ in the second case. Thus, both hypotheses are consistent with the measurements, and we conclude that, in AMC-11, the bare correlation length $\xi_{0}$ is in the range 2 to $5 \AA$.

This result for $\xi_{0}$, which is of the order of magnitude of interatomic distances, contradicts the usual assessment that instead takes $\xi_{0}$ to be of the order of the molecular length. But, in the theory of phase transitions, $\xi_{0}$ is also the basic length of the phases under transition. In this sense, it seems more satisfactory to find it in the range of the rigid elements (the interatomic links), than of the size of the flexible molecules (which have lost their physical individualities).

\section{Conclusion.}

Several independent series of measurements of the tilt susceptibility have been performed at the A-C transition of AMC-11, in a temperature range between $1 \mathrm{mK}$ and $1 \mathrm{~K}$ from the tran- 
sition. All of these measurements give critical values of the exponent $\gamma$ in this range of temperature, consistent with :

$$
\gamma=1.28 \pm 0.03
$$

This result appears to be different from the classical value $(\gamma=1)$, and similar to the helium one $(\gamma=1.31)$ [22]. Such critical behaviour is consistent with the Ginzburg criterion calculated in $\S 3.2$ from our measurement : $\xi_{0} \sim 2-5 \AA$; it is consistent also with the non-classical value of the exponent $\beta$ already found at the A-C transition of AMC-11 $(\beta \sim 0.36)$ [14]. Incidentally, let us note that the determination of these two critical exponents yields the determination of all the other exponents of the transition. In particular, from the relation $\alpha+2 \beta+\gamma=2$, we may deduce the specific-heat exponent $\alpha=0 \pm 0.04$.

Comparisons with the measurements obtained from other experimental methods are instructive also. Our results are consistent with the measurements $\left(\gamma=1.28 ; \xi_{0} \sim 6 \AA\right)$ performed to the precision of $1 \mathrm{mK}$ by $\mathbf{M}$. Delaye [13], but they disagree with the mean-field behaviour found recently by the MIT Group $[8,9]$. One possible explanation for such a discrepancy could be the different families of chemical compounds used by different experimentalists for studying the A-C transition. According to this idea, each family of compounds would have a different $\xi_{0}$, and a different Ginzburg limit, leading to the observation of both mean-field and critical behaviours. But such a possibility seems incompatible with the physical meaning attributed to $\xi_{0}$. As discussed in $\S 3.2, \xi_{0}$ should be intrinsic, of the order of magnitude of a few interatomic distances for all materials. Another possibility which might explain the observed discrepancy in the critical behaviours could be the close vicinity of a tricritical point in the phase diagram [23]. The upper critical dimensionality is then equal to 3, causing mean-field behaviour in some range around the tricritical point. Such a possibility may occur in some chemical compounds, but there is no reason to believe that it should be a general rule.

Though similar to the exponents of the $X Y$-model in 3-dimensions $(\beta=0.346 ; \gamma=1.31)$ [22], the exponents $\beta$ and $\gamma$ measured in AMC-11 appear to be slightly different. One possible explanation for such a difference could come from the complications exhibited by smectics, which are not taken into account in the $X Y$-model, and which do not exist in the superfluid transition. For example, the existence of an anisotropic elastic constant, or the coupling of the order parameter with the Landau-Peierls instability of the smectic layers [2], could produce a slight modification in the critical exponents.

\section{Acknowledgments.}

We are very grateful to G. Durand and P. G. de Gennes for helpful discussions and to P. Keller, L. Liébert and L. Strzelecki for careful chemical synthesis and purifications.

\section{References}

[1] De Gennes, P. G., The Physics of Liquid Crystals (Clarendon, Oxford) 1975.

[2] De Gennes, P. G., J. Physique Colloq. 30 (1969) C4-65.

[3] De Gennes, P. G., C. R. Heb. Séan. Acad. Sci. 274B (1972) 758.

De Gennes, P. G., Mol. Cryst: Liq. Cryst. 21 (1973) 49.

[4] Ginzburg, V. L., Sov. Phys. Solid State 2 (1960) 1824 (Fiz. Tverd. Tela 2, no 9 (1960) 2031).

[5] Delaye, M., Keller, P., Phys. Rev. Lett. 37 (1976) 1065.

[6] Gulllon, D., Skoulios, A., J. Physique 38 (1977) 79.

[7] Мeiboom, S., Hewitt, R. C., Phys. Rev. A 15 (1977) 2444.

[8] Safinya, C. R., Kaplan, M., Als-Nielsen, J., Birgeneau, R. J., Davidov, D., Litster, J. D., JohnSon, D. L., Neubert, M., Phys. Rev. B 21 (1980) 4149. 
[9] Rosenblatt, Ch., Litster, J. D., Phys. Rev. A 26 (1982) 1809.

[10] Wise, R. A., Smith, D. H., Doane, J. W., Phys. Rev. A 7 (1973) 1366.

[11] Flanders, P. J., Appl. Phys. Lett. 28 (1976) 571.

[12] Garoff, S., Meyer, R. B., Phys. Rev. Lett. 38 (1977) 848.

[13] Delaye, M., J. Physique Colloq. 40 (1979) C3-350.

[14] Galerne, Y., Phys. Rev. A 24 (1981) 2284.

[15] Kahn, F. J., Appl. Phys. Lett. 22 (1973) 386.

[16] Galerne, Y., Thèse d'Etat (Orsay, 1983).

[17] Galerne, Y., J. Physique 39 (1978) 1311.

[18] Ho, J. T., Litster, J. D., Phys. Rev. Lett. 22 (1969) 603.

[19] Suter, R. M., Hohenemser, C., J. Appl. Phys. 50 (1979) 1814.

[20] Kleman, M., Williams, C. E., J. Physique Lett. 35 (1974) L-49.

[21] Schantz, C. A., Johnson, D. L., Phys. Rev. A 17 (1978) 1504.

[22] Le Guillou, J. C., Zinn-Justin, J., Phys. Rev. Lett. 39 (1977) 95.

[23] Garland, C. W., private communication. 